\title{
Risk factors of different hemoplasma species infections in cats
}

\author{
Michèle Bergmann ${ }^{1 *}$, Theresa Englert ${ }^{1}$, Bianca Stuetzer ${ }^{1}$, Jennifer R. Hawley², Michael R. Lappin² \\ and Katrin Hartmann ${ }^{1}$
}

\begin{abstract}
Background: Hemoplasma species (spp.) commonly cause infections in cats worldwide. However, data on risk factors for infections are limited. The aim of this study was to determine the prevalence of hemoplasma spp. infections in cats in Southern Germany and to assess risk factors associated with infection.

Results: DNA was extracted from blood samples of 479 cats presented to different veterinary hospitals for various reasons. DNA of feline hemoplasmas was amplified by use of a previously reported PCR assay. Direct sequencing was used to confirm all purified amplicons and compared to hemoplasma sequences reported in GenBank. Results were evaluated in relation to the age, sex, housing conditions, feline leukemia virus (FeLV) and feline immunodeficiency virus (FIV) status of the cats.

The overall hemoplasma prevalence rate was 9.4\% (45/479; 95\% Cl: 7.08-12.36). 'Candidatus Mycoplasma (M.) haemominutum' (Mhm) DNA was amplified from 42 samples, M. haemofelis from 2, and M. haemocanis from 1 sample. There was a significantly higher risk of hemoplasma infection in cats from multi-cat households, in outdoor cats, as well as in cats with FIVinfection and in cats with abortive FeLV infection, but not in cats with progressive or regressive FeLV infection.
\end{abstract}

Conclusions: Mhm infection is common in cats in Southern Germany. Higher prevalence in multi-cat households and associations with FeLV infection likely reflect the potential for direct transmission amongst cats. Outdoor access, male gender, and FIV infection are additional risk factors that might relate to aggressive interactions and exposure to vectors.

Keywords: Feline, Hemoplasmosis, Mycoplasma spp, PCR, Vector-borne, FeLV, FIV

\section{Background}

Hemoplasma species (spp.) (or hemotrophic Mycoplasma spp.) are bacteria without cell walls that can cause hemolytic anemia in different species. At least 3 hemoplasma spp. have been described in cats, Mycoplasma (M.) haemofelis (Mhf), 'Candidatus (Ca.) M. haemominutum' (Mhm), and 'Ca. M. turicensis' (Mtc). Hemoplasma spp. attach to the external surface of red blood cells. Besides transmission through arthropod vectors, there is evidence of horizontal transmission (e.g., blood transmission during aggressive interactions between cats, blood transfusion) between cats [17].

Hemoplasma strains that occur in cats differ in their size and pathogenicity. Mhf and Mtc seem to have a

\footnotetext{
* Correspondence: n.bergmann@medizinische-kleintierklinik.de

${ }^{1}$ Clinic of Small Animal Medicine, Centre for Clinical Veterinary Medicine,

LMU Munich, Veterinaerstrasse 13, 80539 Munich, Germany

Full list of author information is available at the end of the article
}

higher pathogenicity and are more often associated with anemia than $\mathrm{Mhm}$ [2]. However, clinical relevance of hemoplasmas as a cause of anemia is not fully understood. Many authors believe them to be an important primary cause of anemia [20,21, 25]; others regard hemoplasmas more as opportunistic pathogens $[5,12]$.

Natural hemoplasma infection is mostly subclinical. Clinical signs can occur after a longer latency under immunosuppressed conditions. Feline immunodeficiency virus (FIV) and feline leukemia virus (FeLV) infection can be associated with the development of clinical signs in some cats $[4,5,24]$. After developing clinical signs, alternating periods with anemia and subclinical phases can occur $[2,25]$.

Worldwide, cats are commonly infected by hemoplasma spp. [11, 12, 14, 21, 26, 27] and prevalence was found to be $9.9 \%$ in Switzerland [27] and 38.5\% in Africa [14]. In Southern Germany, two studies investigated the 
prevalence of hemoplasma spp. infection in 135 cats [11] and in 296 cats [12] so far. These studies only evaluated preselected cats with anemia. Current data in a non-selected cat population are missing. Thus, the aim of the present study was to determine the prevalence of hemoplasma spp. by investigating blood samples of 479 cats by PCR. In addition, associations between hemoplasma spp. infections with the age, sex, housing conditions, and FIV and FeLV infection status of the cats were evaluated.

\section{Methods}

\section{Animals}

The 479 cats evaluated in this study were presented to different veterinary clinics in Southern Germany for various reasons. Health status of the cats was evaluated by physical examination. In each cat, a complete blood count $(\mathrm{CBC})$ was performed and the FIV and FeLV status was examined. FIV antibodies were detected using a commercial enzyme-linked immunosorbent assay (ELISA) (SNAP Kombi Plus FeLV/FIV antibody test ${ }^{\circ}$, IDEXX GmbH, Ludwigsburg, Germany). FeLV infection status was investigated by performing tests for free FeLV p27 antigen using a commercial ELISA (SNAP Kombi Plus FeLV/FIV antibody test; IDEXX GmbH, Ludwigsburg, Germany), FeLV provirus using polymerase chain reaction (PCR) as well as anti-FeLV-p45 antibodies using an indirect ELISA, both as previously described [1]. Progressively FeLV- infected cats are persistently viremic and thus, FeLV antigen- and proviruspositive [9]. Regressively FeLV-infected cats are antigennegative and provirus-positive; they are considered FeLV carriers. Cats with abortive FeLV infection never become viremic; they are antigen-, and provirus-negative but have FeLV-specific antibodies [9].

A total of 298 cats were male $(62.2 \%)$ and 181 cats were female $(37.8 \%)$ (Table 1$)$. The cats' ages ranged from 3 months to 19 years. Median age was 7.4 years (age of 40 cats was unknown). Of the 468 cats, 106 (22.6\%) were purebred cats and $362(77.4 \%)$ were Domestic Shorthair (DSH). Breed was unknown in 11 (2.3\%) cats. Nine of 479 cats (1.9\%) were progressively FeLV-infected, 7 of 479 cats (1.5\%) were regressively FeLV-infected, and 22 of 479 cats (4.5\%) were abortively FeLV-infected. A total of 7 cats were FIV antibodypositive (1.5\%); two of these $7 \mathrm{FIV}$-infected cats were also progressively FeLV-infected.

\section{Hemoplasma spp. PCR}

Total DNA had previously been extracted from whole blood of the 479 cats using the MagNA Pure LC Total Nucleic Acid Isolation Kit (Roche Diagnostics AG, Rotkreuz, Switzerland) and stored at $-80{ }^{\circ} \mathrm{C}$ until assayed in this study. The samples were thawed at room temperature and

Table 1 Cats with and without hemoplasma species (spp.) infection, and analysis of the risk factors housing conditions, feline immunodeficiency virus (FIV), and feline leukemia virus (FeLV) infection status

\begin{tabular}{|c|c|c|c|c|c|c|c|c|}
\hline & & $n$ & Hemoplasma spp.-positive $^{a}$ & Hemoplasma spp.-negative ${ }^{a}$ & $p$ & $\mathrm{Mhm}^{\mathrm{a}}$ & Mhf $^{a}$ & $M h c^{a}$ \\
\hline Total & 479 & & $45(9.4)$ & $434(90.6)$ & - & $42(93.3)$ & $2(4.5)$ & $1(2.2)$ \\
\hline Age (median) & & 439 & 9.0 years & 7.2 years & - & 9.3 & 17 & 2 \\
\hline Gender & male & 298 & $38(12.8)$ & $260(87.2)$ & 0.001 & $37(97.4)$ & $0(0.0)$ & $1(2.6)$ \\
\hline$(n=470)$ & female & 181 & $7(3.9)$ & $174(96.1)$ & & $5(71.4)$ & $2(28.6)$ & $0(0.0)$ \\
\hline Origin & shelter & 56 & $10(17.9)$ & $56(82.1)$ & 0.104 & $10(100.0)$ & $0(0.0)$ & $0(0.0)$ \\
\hline$(n=479)$ & private & 423 & $35(8.3)$ & $388(91.7)$ & & $32(91.4)$ & $2(5.7)$ & $1(2.9)$ \\
\hline Household & multi-cat & 378 & $44(11.6)$ & $334(88.4)$ & 0.007 & $41(93.2)$ & $2(4.5)$ & $1(2.3)$ \\
\hline$(n=445)$ & single-cat & 67 & $1(1.5)$ & $66(98.5)$ & & $1(100.0)$ & $0(0.0)$ & $0(0.0)$ \\
\hline Access & outdoor & 229 & $37(16.2)$ & $192(83.8)$ & $<0.0001$ & $39(95.1)$ & $2(4.9)$ & $0(0.0)$ \\
\hline$(n=432)$ & indoor & 203 & $4(2.0)$ & $199(98.0)$ & & $3(75.0)$ & $0(0.0)$ & $1(25.0)$ \\
\hline FIV infection & positive & 7 & $5(71.4)$ & $2(28.6)$ & $<0.0001$ & $5(100.0)$ & $0(0.0)$ & $0(0.0)$ \\
\hline$(n=477)$ & negative & 470 & $40(8.5)$ & $430(91.5)$ & & $37(92.5)$ & $2(5.0)$ & $1(2.5)$ \\
\hline Progressive FeLV infection & positive & 9 & $1(11.1)$ & $8(88.9)$ & ns & $1(100.0)$ & $0(0.0)$ & $0(0.0)$ \\
\hline$(n=479)$ & negative & 470 & $44(9.4)$ & $426(91.5)$ & ns & $41(93.2)$ & $2(4.5)$ & $1(2.3)$ \\
\hline Regressive FeLV infection & positive & 7 & $2(28.6)$ & $5(71.4)$ & ns & $2(100.0)$ & $0(0.0)$ & $0(0.0)$ \\
\hline$(n=479)$ & negative & 472 & $43(9.1)$ & $429(90.9)$ & ns & $40(93.0)$ & $2(4.7)$ & $1(2.3)$ \\
\hline Abortive FeLV infection & positive & 22 & $12(54.5)$ & $10(45.5)$ & $<0.0001$ & $12(100.0)$ & $0(0.0)$ & $0(0.0)$ \\
\hline$(n=459)$ & negative & 437 & $28(6.4)$ & 409 (93.6) & & $23(82.2)$ & $2(7.2)$ & $1(3.6)$ \\
\hline
\end{tabular}

$n$ numbers of cats in every group, $p$ p-value, $n s$ not significant, Mhm 'Candidatus Mycoplasma (M.) haemominutum', Mhf M. haemofelis, Mhc M. haemocanis; ${ }^{a}$ the percentages are presented in parenthesis 
prepared for the amplification of hemotropic Mycoplasma spp. DNA in a previously reported conventional polymerase chain reaction (PCR) assay [10]. Briefly, each $2.5 \mu \mathrm{l}$ DNA sample was added to the PCR master mix $(10 \mathrm{mM}$ Tris, $\mathrm{pH}$ 8.3, $50 \mathrm{mM} \mathrm{KCl}, 3.5 \mathrm{mM} \mathrm{MgCl} 2,200 \mu \mathrm{M}$ each dNTP, $400 \mu \mathrm{M}$ dUTP, 0.5 units uracil N-glycosylase (UNG) and 1.25 units Taq polymerase) with the final reaction volume of $25 \mu$ l.

PCR products were visualized on a $3 \%$ agarose gel using 6X EZVision One DNA Dye (Amersco; Solon, $\mathrm{OH}$ ) according to manufacturer specifications.

Appropriate negative and positive controls were run with each sample. Positive PCR controls were obtained from diagnostic whole blood samples received by the Center for Companion Animal Studies (Department of Clinical Sciences, Colorado State University). Negative controls consisted of PCR (molecular grade) water being added in lieu of DNA template. Any positive sample was purified (QIAquick Gel Extraction Kit; Qiagen Germantown, MD) and sequenced to confirm genus and species (Colorado State University Proteomics and Metabolomics Facility; Fort Collins Colorado).

\section{Statistical analysis}

Statistical analysis was performed with Graph PadPrism 6.0. Confidence intervals were determined by an exact binomial test. The exact binomial test was one-tailed and was used to prove the alternative hypotheses that the prevalence of hemoplasma infection was within the 95\% confidence interval (CI). A significance level of $<0.05$ was chosen. Fisher's exact test was used to assess associations between hemoplasma infection, cats' age, gender, and housing conditions, as well as FIV and FeLV status.

\section{Results}

\section{Prevalence of hemoplasma spp. infections}

The overall hemoplasma prevalence rate was 9.4\% (45/ 479; 95\% CI: 7.08-12.36) (Table 1). Mhm DNA was amplified from 42 samples, Mhf from 2 samples, and Mycoplasma hemocanis (Mhc) from 1 sample (Table 1). All PCR-negative and extract-negative controls were always negative in all assays.

\section{Hemoplasma-infected cats}

Of the hemoplasma-positive cats, $84.5 \%$ (38/45) were male. Infected cats ages ranged from 9 months to 17 years (median 9 years). Most of the hemoplasma-infected cats were $\geq 9$ years old (25/41); 8 cats were $\leq 3$ years old (age of 4 cats was unknown).

Most of the infected cats were allowed to roam outside $(90.2 \% ; 37 / 41)$ and lived in multi-cat-households $(97.8 \%$; 44/45) (Table 1).

Ten of the infected cats were healthy and presented for neutering and/or routine health checks $(22.2 \%$; 10 /
45); the remaining cats were presented with a history of illness $(77.8 \%$; 35/45). Six infected cats had anemia (13.4\%; 6/45), whereas $67(21.1 \%$; 67/317) of the noninfected cats had anemia. The hematocrit of the 6 hemoplasma-infected anemic cats ranged from 0.22 to $0.29 \mathrm{l} / \mathrm{l}$ (reference range: $0.30-0.44 \mathrm{l} / \mathrm{l}$ ). None of these cats showed signs of regenerative anemia, such as polychromasia, macrocytosis, and in none of these cats nucleated red blood cells were present.

\section{Risk factors}

Due to the low number of cats infected with the species Mhf or Mhc, all of the hemoplasma-infected cats were evaluated together for risk factor analysis. Male cats had a significantly higher risk of infection with a hemoplasma spp. than female cats ( $p=0.001$; odds-ratio (OR): 3.633; 95\% CI: 1.586-8.322) (Table 1). In addition, cats with outdoor access $(p<0.0001$; OR: 9.59; 95\% CI: 3.3527.42) and cats from multi-cat households $(p=0.007$; OR: 8.66 ; 95\% CI: 1.18-64.25) had a significantly higher risk of infection with a hemoplasma spp. Cats from shelters $(p=0.104$; OR: 1.98 ; $95 \%$ CI: $92.8-$ 421.9) had no significantly higher risk of hemoplasma-infection than cats from private homes.

Of the hemoplasma-positive cats, 5 cats were coinfected with FIV; 12 cats were abortively FeLV-coinfected, 2 cats were regressively and 1 cat was progressively coinfected with FeLV. Hemoplasma spp. bacteremia was significantly more often found in FIV-positive cats $(p<$ 0.0001; OR: 26.88; 95\% CI: 5.05-143.00) and in cats with abortive FeLV infection ( $p=0.019$; OR: $2.64 ; 95 \%$ CI: $1.26-5.51)$. Cats with progressive $(p=0.592)$ and regressive $(p=0.134)$ FeLV infections had no higher risk of being infected with hemoplasma spp..

\section{Mhc-infected cat}

The Mhc-infected cat was a 10 months old, female neutered British Shorthair. The cat lived in a multi-cat household. The cat had no access to outdoors, no contact to dogs, and no travelling history. The cat was presented for routine health care. Physical and laboratory examination were unremarkable.

\section{Discussion}

The present study revealed an overall prevalence rate of hemoplasma infections in cats of 9.4\%. Worldwide, prevalence differs between about $10 \%$ in Switzerland [27] to over $30 \%$ in Africa [14]. Thus, prevalence of hemoplasma infection seems to be higher in countries with warmer climates. The infection rate in Southern Germany is similar to that in Switzerland, presumably due to the similar climate. An association between prevalence of potential vectors and prevalence of feline hemoplasma infections is likely $[18,23]$. 
Similar to previous studies, Mhm was the most common hemoplasma spp. in the present study. None of the cats was positive for Mtc, although Mtc infections in cats from Southern Germany have been reported previously [11, 12]. Surprisingly, a 10 months old British Short Hair cat was infected with Mhc, the most prevalent hemoplasma spp. in dogs. Hemoplasmas are not necessarily strictly species-specific and there are reports stating that Mhc could infect cats and that cats might act as reservoir for Mhc $[15,16]$. Mhc is most prevalent in dogs from Mediterranean countries; transmission is suspected via Rhipicephalus sanguineus, a tick that is not endemic in Germany [22, 28]. Source of Mhc infection in this cat of the present study is unclear. The cat was never allowed to go out and lived only indoors without any contact to dogs. Tick transmission therefore seemed unlikely; as this cat was still young, vertical transmission, which has been reported in cattle, might be the most likely source of infection [3].

Most of the Mhm- and Mhf-infected cats were older ( $\geq 9$ years). Due to the subclinical nature of $\mathrm{Mhm}$ infection, this follows the presumption of becoming infected as a result of prolonged exposure during lifetime $[23,27]$.

As reported previously, infection was more prevalent in male cats and in cats with outdoor access [27]. This supports the hypotheses of horizontal transmission during aggressive interactions between cats and transmission through arthropod vectors [17]. In addition, cats from multicathouseholds were significantly more likely to be infected with hemoplasma spp., which could be due to a higher risk for aggressive interactions in multicat-environments.

Associations between hemoplasma spp. infection and clinical disease in naturally infected cats are not fully understood. Natural hemoplasma spp. infection is mainly subclinical in cats, but some cats develop severe anemia with fever, inappetence and lethargy [21]. It has been discussed whether retrovirus and hemoplasma infections are associated and whether immunosuppression could lead to enhanced pathogenicity of hemoplasma spp. [8, 23, 27].

A surprisingly strong association of FIV and hemoplasma spp. infection was found in the present study. Cats with hemoplasma infection were 26 times more likely to be FIV infected. This emphasizes, that hemoplasma spp. can be transmitted through similar routes as FIV, such as through biting. Similar to a recent study, FIV infection was predominantly associated with Mhm but not with Mhf and Mtc [23]. However, none of the hemoplasma FIV-coinfected cats showed signs of clinical manifestation of hemoplasmosis. It has been discussed that specific FIV strains might be the reason for more severe disease in coinfected cats [5], but this has never been proven so far.
In the present study, cats with hemoplasma infection were also more likely to be FeLV-infected. This is presumably attributable to outdoor access and multi-cat household, which increases the risk of FeLV infection through cat contact [7]. Only 3 of 28 FeLV hemoplasma-coinfected cats showed mild non-regenerative anemia which was likely caused by chronic kidney disease present in these 3 cats. In contrast, Harrus and colleagues found that Mhfpositive and cats positive for FeLV-p27 antigen were more often anemic than cats with Mhf infection alone [8]. However, most of the hemoplasma-positive cats in the present study were only abortively FeLV-infected (12/45), and cats with abortive FeLV infection never become viremic [9]. Abortive infections develop in immunocompetent cats. Due to effective immune response, these cats are able to terminate viral replication, and cats have no clinical signs [9]. Thus, immunocompetence is likely the reason for the missing enhanced pathogeniticity of hemoplasmas in the cats with abortive FeLV coinfections. In addition, all of the FeLV hemoplasma-coinfected cats were infected with Mhm, a strain that has an inherently low pathogenicity [2]. None of the progressively and regressively FeLV hemoplasma-coinfected cats showed clinical signs either. All this supports the hypothesis that the association of FIV and FeLV with hemoplasma spp. infection is likely the same mode of transmission and not necessarily an enhanced pathogenicity.

If hemoplasma spp. infection is clinically apparent, it usually results in regenerative hemolytic anemia [19]. In the present study, only $6 \mathrm{Mhm}$-infected of all 45 hemoplasmainfected cats had anemia. All of these cats had nonregenerative anemia, and other diseases that were likely responsible for the non-regenerative anemia (chronic kidney disease $(n=3)$, anemia of chronic disease caused by chronic respiratory tract disease $(n=2)$; tumor of the central nervous system $(n=1)$; chronic weight loss $(n=1))$. Thus, other reasons than Mhm infection were presumably responsible for anemia in these cats. Although Mhf is thought to be more pathogenic, none of the Mhf-infected cats in the present study showed clinical signs. This could be due to a low hemoplasma load or infection with a less virulent strain. The Mhc-infected cat also showed no clinical signs of hemoplasmosis. Pathogenicity of this species in cats is unknown. Mhc can cause acute disease in immunosuppressed [6] or splenectomized dogs [13].

The major limitation of the study was the low number of cats with Mhf and Mhc infections and the fact that no cat in the present study was positive for Mtc. Thus, comparison between the different hemoplasma species was not possible. In addition, pretreatment of the cats in the present study was unknown, and treatment with antibiotics, e.g., doxycycline or enrofloxacin, prior to blood sampling could have led to termination of bacteremia and underestimation of hemoplasma prevalence [21]. 


\section{Conclusions}

Feline hemoplasma infection is relatively common in cats, and if cats are infected, they are usually infected with Mhm, a species with low pathogenicity. A higher risk exists for male sex, outdoor access, multi-cat environment, and FIV- and FeLV-infected cats which seems to be related to similar mechanisms for transmission during aggressive interactions and through arthropod vectors.

\section{Abbreviations \\ Ca.: Candidatus; CBC: Complete blood count; DSH: Domestic Shorthair: ELISA: Enzyme-linked immunosorbent assay; FeLV: Feline leukemia virus; FIV: Feline immunodeficiency virus; M.: Mycoplasma; Mhc: Mycoplasma haemocanis; Mhf: Mycoplasma haemofelis; Mhm: 'Candidatus Mycoplasma haemominutum'; Mtc: 'Candidatus Mycoplasma turicensis'; n: Numbers of cats in every group; ns: Not significant; $\mathrm{p}$ : P-value; PCR: Polymerase chain reaction; spp.: Species}

\section{Acknowledgements}

The authors would like to thank Prof. Dr. Ralf S. Mueller, Clinic of Small Animal Medicine, for his assistance in the statistical examination.

Parts of the results were presented as a poster at the ACVIM Symposium in San Francisco, USA, 2014.

\section{Funding}

This research received no grant from any funding agency in the public or commercial sectors but was supported in part by the Center for Companion Animal Studies, a non-profit organization, at Colorado State University.

\section{Availability of data and materials}

Raw data (Excel file) is available from the corresponding author (Dr. Michèle Bergmann) upon request.

\section{Authors' contributions}

$\mathrm{KH}$ chaired the research group. Acquisition of data was performed by TE under the supervision of $\mathrm{KH}$. PCR was performed by BS and $\mathrm{JH}$ under the supervision of $\mathrm{ML}$ at the Veterinary Diagnostic Laboratories, Colorado State University. MB did the data analysis and wrote the paper. All authors read, commented, and approved the final manuscript.

\section{Competing interests}

The authors declare that they have no competing interests.

\section{Consent for publication}

Not applicable.

\section{Ethics approval}

The study was performed on stored blood samples that had been collected for the purpose of a previous study with the agreement of cat owners (including the director and veterinary services of local shelters) that presented their cats to different veterinary clinics in Southern Germany for various reasons. As stored blood samples were used, no seperat ethical approval was required for the study.

\section{Author details}

${ }^{1}$ Clinic of Small Animal Medicine, Centre for Clinical Veterinary Medicine, LMU Munich, Veterinaerstrasse 13, 80539 Munich, Germany. ${ }^{2}$ Center for Companion Animal Studies, Department of Clinical Sciences, College of Veterinary Medicine and Biomedical Sciences, Colorado State University, Fort Collins, CO, USA

Received: 21 September 2016 Accepted: 17 January 2017

Published online: 16 February 2017

\section{References}

1. Englert T, Lutz H, Sauter-Louis C, Hartmann K. Survey of the feline leukemia virus infection status of cats in Southern Germany. J Feline Med Surg. 2012;14:392-8.
2. Foley JE, Harrus S, Poland A, Chomel B, Pedersen NC. Molecular, clinical, and pathologic comparison of two distinct strains of Haemobartonella felis in domestic cats. Am J Vet Res. 1998;59:1581-8.

3. Fujihara Y, Sasaoka F, Suzuki J, Watanabe Y, Fujihara M, Ooshita K, Ano H, Harasawa R. Prevalence of hemoplasma infection among cattle in the western part of Japan. J Vet Med Sci. 2011;73:1653-5.

4. Gentilini F, Novacco M, Turba ME, Willi B, Bacci ML, Hofmann-Lehmann R. Use of combined conventional and real-time PCR to determine the epidemiology of feline haemoplasma infections in northern Italy. J Feline Med Surg. 2009;11:277-85.

5. George JW, Rideout BA, Griffey SM, Pedersen NC. Effect of pre-existing FeLV infection or FeLV and feline immunodeficiency virus coinfection on pathogenicity of the small variant of Haemobartonella felis in cats. Am J Vet Res. 2002;63:1172-8.

6. Handcock WJ. Clinical haemobartonellosis associated with use of corticosteroid. Vet Rec. 1989;125:585

7. Hardy Jr WD, Hirshaut Y, Hess P. Detection of the feline leukemia virus and other mammalian oncornaviruses by immunofluorescence. Bibl Haematol. 1973;39:778-99.

8. Harrus S, Klement E, Aroch I, Stein T, Bark H, Lavy E, Mazaki-Tovi M, Baneth G. Retrospective study of 46 cases of feline haemobartonellosis in Israel and their relationships with FeLV and FIV infections. Vet Rec. 2002;151:82-5.

9. Hartmann K. Clinical aspects of feline retroviruses: a review. Viruses. 2012;4: 2684-710.

10. Jensen WA, Lappin MR, Kamkar S, Reagan W. Use of a polymerase chain reaction assay to detect and differentiate two strains of Haemobartonella felis in naturally infected cats. Am J Vet Res. 2001;62:604-8.

11. Just F, Pfister K. Detection frequency of haemoplasma infections of the domestic cat in Germany. Berl Munch Tierarztl Wochenschr. 2007;120:197-201.

12. Laberke S, Just F, Pfister K, Hartmann K. Prevalence of feline haemoplasma infection in cats in Southern Bavaria, Germany, and infection risk factor analysis. Berl Munch Tierarztl Wochenschr. 2010;123:42-8.

13. Lester SJ, Hume JB, Phipps B. Haemobartonella canis infection following splenectomy and transfusion. Can Vet J. 1995:36:444-5.

14. Lobetti RG, Tasker S. Diagnosis of feline haemoplasma infection using a realtime PCR assay. J S Afr Vet Assoc. 2004:75:94-9.

15. Lumb W. Canine haemobartonellosis and its feline counterpart. Calif Vet. 1961;14:24-5.

16. Luria BJ, Levy JK, Lappin MR, Breitschwerdt EB, Legendre AM, Hernandez JA, Gorman SP, Lee IT. Prevalence of infectious diseases in feral cats in Northern Florida. J Feline Med Surg. 2004;6:287-96.

17. Museux K, Boretti FS, Willi B, Riond B, Hoelzle K, Hoelzle LE, Wittenbrink MM, Tasker S, Wengi N, Reusch CE, Lutz H, Hofmann-Lehmann R. In vivo transmission studies of 'Candidatus Mycoplasma turicensis' in the domestic cat. Vet Res. 2009;40:45.

18. Novacco M, Meli ML, Gentilini F, Marsilio F, Ceci C, Pennisi MG, Lombardo G, Lloret A, Santos L, Carrapico T, Willi B, Wolf G, Lutz H, Hofmann-Lehmann R. Prevalence and geographical distribution of canine hemotropic mycoplasma infections in Mediterranean countries and analysis of risk factors for infection. Vet Microbiol. 2009;142:276-84.

19. Pennisi MG, Hartmann K, Addie DD. Blood transfusion in cats ABCD guidelines for minimizing risk of infectious iatrogen complications. J Feline Med Surg. 2015:17:588-93.

20. Piros des Santes A, de Oliveira Conrado F, Messick JB, Biondo AW, Tostes de Oliviera S, Sa Guimarares AM, Cannes do Nascimento N, Pedralli V, Lasta CS, Doiaz Gonzales FH. Hemplasma prevalence and hematological abnormalities associated with infection in three different cat populations from Southern Brazil. Braz J Vet Parasitol. 2014;23:428-34.

21. Reynolds CA, Lappin MR. "Candidatus Mycoplasma haemominutum" infections in 21 client-owned cats. J Am Anim Hosp Assoc. 2007:43:249-57.

22. Seneviratna P, Weerasinghe, Ariyadasa S. Transmission of Haemobartonella canis by the dog tick, Rhipicephalus sanguineus. Res Vet Sci. 1973;14:112-4.

23. Sykes JE, Drazenovich NL, Ball LM, Leutenegger CM. Use of conventional and real-time polymerase chain reaction to determine the epidemiology of hemoplasma infections in anemic and nonanemic cats. J Vet Intern Med. 2007;21:685-93.

24. Sykes JE, Terry JC, Lindsay LL, Owens SD. Prevalences of various hemoplasma species among cats in the United States with possible hemoplasmosis. J Am Vet Med Assoc. 2008;232:372-9.

25. Weingart C, Tasker S, Kohn B. Infection with haemoplasma species in 22 cats with anemia. J Feline Med Surg. 2016;18:129-36. 
26. Willi B, Tasker S, Boretti FS, Doherr MG, Cattori V, Meli ML, Lobetti RG, Malik R, Reusch CE, Lutz H, Hofmann-Lehmann R. Phylogenetic analysis of "Candidatus Mycoplasma turicensis" isolates from pet cats in the United Kingdom, Australia, and South Africa, with analysis of risk factors for infection. J Clin Microbiol. 2006;44:4430-5.

27. Willi B, Boretti FS, Baumgartner C, Tasker S, Wenger B, Cattori V, Meli ML, Reusch CE, Lutz H, Hofmann-Lehmann R. Prevalence, risk factor analysis, and follow-up of infections caused by three feline hemoplasma species in cats in Switzerland. J Clin Microbiol. 2006;44:961-9.

28. Willi B, Novacco M, Meli M, Wolf-Jäckel G, Boretti F, Wengi N, Lutz $H_{\text {, }}$ Hofmann-Lehmann R. Haemotropic mycoplasmas of cats and dogs: transmission, diagnosis, prevalence and importance in Europe. Schweiz Arch Tierheilkd. 2010;152:237-44.

Submit your next manuscript to BioMed Central and we will help you at every step:

- We accept pre-submission inquiries

- Our selector tool helps you to find the most relevant journal

- We provide round the clock customer support

- Convenient online submission

- Thorough peer review

- Inclusion in PubMed and all major indexing services

- Maximum visibility for your research

Submit your manuscript at www.biomedcentral.com/submit
Biomed Central 